\title{
A Variation of the $q$-Painlevé System with Affine Weyl Group Symmetry of Type $E_{7}^{(1)}$
}

\author{
Hidehito NAGAO
}

Department of Arts and Science, National Institute of Technology, Akashi College, Hyogo 674-8501, Japan

E-mail:nagao@akashi.ac.jp

Received July 03, 2017, in final form November 24, 2017; Published online December 10, 2017 https://doi.org/10.3842/SIGMA.2017.092

\begin{abstract}
Recently a certain $q$-Painlevé type system has been obtained from a reduction of the $q$-Garnier system. In this paper it is shown that the $q$-Painlevé type system is associated with another realization of the affine Weyl group symmetry of type $E_{7}^{(1)}$ and is different from the well-known $q$-Painlevé system of type $E_{7}^{(1)}$ from the point of view of evolution directions. We also study a connection between the $q$-Painlevé type system and the $q$-Painlevé system of type $E_{7}^{(1)}$. Furthermore determinant formulas of particular solutions for the $q$-Painlevé type system are constructed in terms of the terminating $q$-hypergeometric function.
\end{abstract}

Key words: $q$-Painlevé system of type $E_{7}^{(1)} ; q$-Garnier system; Padé method; $q$-hypergeometric function

2010 Mathematics Subject Classification: 14H70; 33D15; 33D70; 34M55; 37K20; 39A13; $41 \mathrm{~A} 21$

\section{Introduction}

\subsection{Background}

In [39] H. Sakai has classified the second order continuous and discrete Painlevé equations into 22 cases by using the geometric theory of certain rational surfaces, called the"spaces of initial values" ${ }^{1}$, connected to affine root systems. The spaces of initial values are obtained from $\mathbb{P}^{1} \times \mathbb{P}^{1}$ (resp. $\mathbb{P}^{2}$ ) by blowing up at 8 (resp. 9) singular points. In view of the configuration of 8 (resp. 9) singular points in $\mathbb{P}^{1} \times \mathbb{P}^{1}$ (resp. $\mathbb{P}^{2}$ ), there exist three types of discrete Painlevé equations and six continuous Painlevé equations in the classification: elliptic difference $(e-)$, multiplicative difference $(q-)$, additive difference $(d-)$ and continuous (differential). Each of these Painlevé equations is constructed in a unified manner as the bi-rational action of a translation part of the corresponding affine Weyl group symmetry on a certain family of the rational surfaces. The sole $e$-Painlevé equation [31] having the affine Weyl group symmetry of type $E_{8}^{(1)}$ is obtained from the most generic configuration on the unique curve of bi-degree $(2,2)$ called the smooth "elliptic curve". All of the other Painlevé equations are derived from its degeneration. For instance, the $q$-Painlevé system with the symmetry of type $E_{7}^{(1)}$ is well known to be obtained from a configuration of eight singular points on two curves of bi-degree $(1,1)$ in $\mathbb{P}^{1} \times \mathbb{P}^{1}$. The second order continuous and discrete Painlevé equations are classified into the 22 cases $^{2}$ according to the degeneration diagram of affine Weyl group symmetries (see Fig. 1), where the symbol $A \rightarrow B$ represents that $B$ is obtained from $A$ by a certain limiting procedure. The $d$-Painlevé equation of type $D_{4}^{(1)}$ and its degeneration (expect for $A_{0}^{(1)}$ ) arise as Bäcklund (Schlesinger)

\footnotetext{
${ }^{1}$ For each of the six continuous Painlevé equations, K. Okamoto has constructed certain rational surfaces, called the "spaces of initial values", which parametrize all the solutions [34].

${ }^{2}$ Some $q$-Painlevé equations, such as a second order case of the system [15] (see also [45]), does not belong to the list of discrete Painlevé equations appeared in [39].
} 


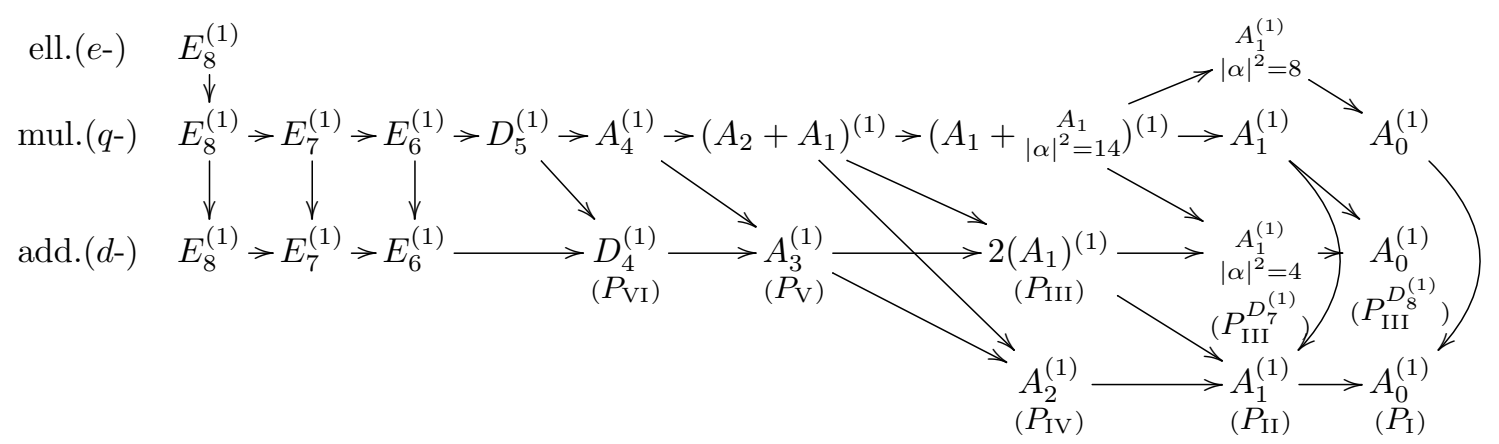

Figure 1 .

transformations of the six continuous Painlevé equations ${ }^{3}\left(P_{\mathrm{I}}, \ldots, P_{\mathrm{VI}}\right)$. The symbol $\begin{aligned} & A_{1}^{(1)} \text { means } \\ & |\alpha|^{2}=l\end{aligned}$ the root subsystem of type $A_{1}^{(1)}$ whose square length of roots is $l$.

Similarly to the differential Painlevé systems, the discrete Painlevé systems are known to have particular solutions expressed by various hypergeometric functions $[12,13,14,16,21,22$, 38]. The particular solutions of the elliptic Painlevé equation are expressed in [12] in terms of the elliptic hypergeometric function ${ }_{10} E_{9}$ [3]. In the case of $q-E_{7}^{(1)}$, the particular solutions are expressed in [24] in terms of the terminating $q$-hypergeometric function ${ }_{4} \varphi_{3},{ }^{4}$ where the function $k \varphi_{l}[3]$ is defined by

$$
{ }_{k} \varphi_{l}\left(\begin{array}{ccc}
\alpha_{1}, & \ldots, & \alpha_{k} \\
\beta_{1}, & \ldots, & \beta_{l}
\end{array}, x\right)=\sum_{s=0}^{\infty} \frac{\left(\alpha_{1}, \ldots, \alpha_{k}\right)_{s}}{\left(\beta_{1}, \ldots, \beta_{l}, q\right)_{s}}\left[(-1)^{s} q^{\left(\begin{array}{c}
s \\
2
\end{array}\right)}\right]^{1+l-k} x^{s}
$$

with $\left(\begin{array}{l}s \\ 2\end{array}\right)=\frac{s(s-1)}{2}$. Here the standard $q$-Pochhammer symbol ${ }^{5}$ is defined by

$$
(x)_{\infty}:=\prod_{i=0}^{\infty}\left(1-q^{i} x\right), \quad(x)_{s}:=\frac{(x)_{\infty}}{\left(x q^{s}\right)_{\infty}}, \quad\left(x_{1}, x_{2}, \ldots, x_{k}\right)_{s}:=\left(x_{1}\right)_{s}\left(x_{2}\right)_{s} \cdots\left(x_{k}\right)_{s}
$$

It is common to nonlinear integrable systems that they arise as the compatibility condition of linear equations and their deformed equations. The pair of the linear equations is called a "Lax pair" for the nonlinear system. Similarly to the continuous Painlevé equations [8, 9, 10, 32, 33], Lax pairs for the discrete Painlevé equations have been studied from various points of view in $[4,11,16,23,37,42,46,48]$. For instance, as a geometric approach, the Lax pair for the $e$-Painlevé equation has been formulated in [46] as a curve of bi-degree $(3,2)$ in $\mathbb{P}^{1} \times \mathbb{P}^{1}$ passing through 12 points. In the case of $q-E_{7}^{(1)}$, the Lax pair has been similarly formulated in $[16,46]$.

In [41] the $q$-Garnier system was formulated as a multivariable extension of the well-known $q-P_{\mathrm{VI}}$ (i.e., $\left.q-D_{5}^{(1)}\right)$ system [11] by H. Sakai, and has recently been studied in $[28,35,40]^{6}$. In [28] a Lax pair, an evolution equation and two kinds of particular solutions ${ }^{7}$ for the $q$-Garnier system have been simply expressed by applying a certain method of Padé approximation and

\footnotetext{
${ }^{3} P_{\text {III }}^{D_{i}^{(1)}}$ symbolizes $P_{\text {III }}$ having the surface connected to the affine root system of type $D_{i}^{(1)}$.

${ }^{4}$ The terminating balanced ${ }_{4} \varphi_{3}$ is rewritten into the terminating $q$-hypergeometric function ${ }_{8} W_{7}$ by Watson's transformation formula [3]. For particular solutions in terms of ${ }_{8} W_{7}$, see [13, 14, 21].

${ }^{5}$ Actually Pochhammer himself used the symbol $(a)_{n}$ not as a rising shifted factorial but as a binomial coefficient [17].

${ }^{6}$ For the related works, see [1, 2, 35] (additive Garnier system), [36, 50] (elliptic Garnier system).

${ }^{7}$ These solutions have been constructed in terms of the $q$-Appell Lauricella function (resp. the generalized $q$-hypergeometric function) in [28, 40] (resp. [28]).
} 
its analogue (i.e., Padé interpolation $)^{8}$. The $q-D_{5}^{(1)}\left(\right.$ resp. $\left.q-E_{6}^{(1)}\right)$ system appears as a reduction of case $N=1$ [41] (resp. particular case of $N=2$ [42]) of the $q$-Garnier system having $2 N$ dependent variables. Recently the $q$-Painlevé type system ${ }^{9}$ [28, Section 2.5] has appeared as a particular case of $N=3$. However the $q-E_{8}^{(1)}$ system has been not obtained from a reduction of the $q$-Garnier system.

Remark 1.1. We call a certain $q$-Painlevé type system "a variation of a q-Painlevé system" having a well-known direction ${ }^{10}$, when both systems satisfy the following: (i) They are associated with different realizations of the symmetry/surface of the same type in the Sakai's classification. (ii) Their time evolutions are different from the viewpoint of shift operator on parameters.

\subsection{Purpose and organization}

Our main subject is the $q$-Painlevé type system [28] regarded as a variation of the well-known $q-E_{7}^{(1)}$ system [5]. The purpose of this paper is the following three.

- We show that the $q$-Painlevé type system is a bi-rational transformation and is related to a novel realization of the symmetry/surface of type $E_{7}^{(1)} / A_{1}^{(1)}$. Then it is clarified to be a variation of the $q-E_{7}^{(1)}$ system.

- The Lax pair for the $q$-Painlevé type system is obtained from a certain reduction of the $q$-Garnier system and we study a connection between the $q$-Painlevé type system and the $q-E_{7}^{(1)}$ system by comparing their Lax equations.

- Particular solutions for the $q$-Painlevé type system are given as a reduction of the $q$-Garnier system.

This paper is organized as follows. In Section 2 we prove that the $q$-Painlevé type system is a bi-rational transformation and we investigate its configuration of 8 singular points on a curve of bi-degree $(2,2)$ in coordinates $(f, g) \in \mathbb{P}^{1} \times \mathbb{P}^{1}$. In Section 3 we briefly recall Lax equations for the $q$-Garnier system [28, Section 2.1], and study a reduction of particular case $N=3$ of the $q$-Garnier system. Consequently, we obtain Lax equations for the $q$-Painlevé type system. In Section 4 the Lax equation for the $q$-Painlevé type system is uniquely determined by a characterization, and recall the characterization of Yamada's Lax equation for the $q-E_{7}^{(1)}$ system. Then we investigate a connection between these systems by comparing characterizations of their Lax equations. In Section 5 we recall the particular solutions of the $q$-Garnier system and we construct particular solutions of the $q$-Painlevé type system by applying a reduction. In Appendix A we derive the $q$-Painlevé type system, its Lax pair and its particular solutions by using a Padé interpolation.

\section{$2 \quad q$-Painlevé type system}

In this section we first recall the $q$-Painlevé type system [28, Section 2.5]. Then we prove that the system is a bi-rational transformation and confirm that the system has the symmetry/surface of type $E_{7}^{(1)} / A_{1}^{(1)}$ by its configuration of eight singular points. Let $q(|q|<1), a_{1}, \ldots, a_{4}, b_{1}, \ldots, b_{4}$, $c_{1}$ and $d_{1} \in \mathbb{C}^{\times}$be complex parameters with a constraint $\prod_{i=1}^{4} \frac{a_{i}}{b_{i}}=q \frac{c_{1}^{2}}{d_{1}^{2}}$, and let $(f, g) \in \mathbb{P}^{1} \times \mathbb{P}^{1}$ be

\footnotetext{
${ }^{8}$ The Padé method has been also applied to the continuous/discrete Painlevé systems in [6, 24, 25, 26, 27, 30, 47, 49]. For the case of $q-E_{7}^{(1)}$ [24], see Section A. For the works related to the differential Garnier system, see [18, 47] (Padé approximation), [19, 20] (Hermite-Padé approximation).

${ }^{9}$ As another derivation of the equations (2.2) and (3.6), see Appendix A.1 (Padé interpolation method).

${ }^{10}$ In case of $q-E_{7}^{(1)}, T_{2}(4.7)$ is the well-known direction and $T_{1}(2.1)$ is a variation direction.
} 
dependent variables. Define $T_{a}: a \rightarrow q a$ by a $q$-shift operator of parameter $a$. Then we consider a $q$-shift operator $T_{1}{ }^{11}$

$$
T_{1}=T_{a_{1}}^{-1} T_{b_{1}}^{-1} .
$$

Here for any object $X$ the corresponding shifts are denoted as $\bar{X}:=T_{1}(X)$ and $\underline{X}:=T_{1}^{-1}(X)$. The operator $T_{1}$ plays the role of the evolution of the system. The system is described by the following transformation $T_{1}^{-1}(g)=\underline{g}(f, g)$ and $T_{1}(f)=\bar{f}(f, g)$ in $\mathbb{P}^{1} \times \mathbb{P}^{1}$ :

$$
\begin{aligned}
& \left(e_{1} f^{2}+e_{1} f g+c_{1}\right)\left(\frac{e_{1}}{q} f^{2}+\frac{e_{1}}{q} g f+c_{1}\right)=c_{1}^{2} \frac{\prod_{i=2}^{4}\left(1-a_{i} f\right)\left(1-b_{i} f\right)}{\left(1-a_{1} f\right)\left(1-b_{1} f\right)}, \\
& \frac{x_{1}^{2}\left(1-f x_{1}\right)\left(1-\bar{f} x_{1}\right)}{x_{2}^{2}\left(1-f x_{2}\right)\left(1-\bar{f} x_{2}\right)}=\prod_{i=2}^{4} \frac{\left(x_{1}-a_{i}\right)\left(x_{1}-b_{i}\right)}{\left(x_{2}-a_{i}\right)\left(x_{2}-b_{i}\right)} .
\end{aligned}
$$

Here $e_{1}=d_{1} a_{2} a_{3} a_{4} b_{1}^{-1}$, and $x=x_{1}, x_{2}\left(=\frac{e_{1}}{c_{1} x_{1}}\right)$ are solutions of an equation $\varphi=0$ where

$$
\varphi(x)=e_{1}+e_{1} g x+c_{1} x^{2} .
$$

Then we have

Proposition 2.1. The q-Painlevé type system (2.2) has the following properties:

(i) It is a bi-rational transformation $T_{1}^{-1}(g)=\underline{g}(f, g)$ and $T_{1}(f)=\bar{f}(f, g) \in \mathbb{P}^{1} \times \mathbb{P}^{1}$.

(ii) It is associated with a novel realization (2.5) of the symmetry/surface of type $E_{7}^{(1)} / A_{1}^{(1)}$.

Proof. (i) It is easy to see that the first equation of (2.2) is a rational transformation $T_{1}^{-1}(g)=$ $g(f, g)$. The second equation of $(2.2)$ is rewritten as

$$
\bar{f}=\frac{x_{1}^{2}\left(1-f x_{1}\right) \mathcal{A}\left(x_{2}\right)-x_{2}^{2}\left(1-f x_{2}\right) \mathcal{A}\left(x_{1}\right)}{x_{1}^{3}\left(1-f x_{1}\right) \mathcal{A}\left(x_{2}\right)-x_{2}^{3}\left(1-f x_{2}\right) \mathcal{A}\left(x_{1}\right)},
$$

where $\mathcal{A}(x)=\prod_{i=2}^{4}\left(x-a_{i}\right)\left(x-b_{i}\right)$. Then the numerator and denominator of (2.4) are alternating with respect to $x_{1} \leftrightarrow x_{2}=\frac{e_{1}}{c_{1} x_{1}}$, and Laurent polynomials $\sum_{i=-4}^{4} h_{i} x_{1}^{i}$ where $h_{i}$ is depending on $f, a_{i}, b_{j}, c_{1}$ and $d_{1}$. Accordingly the numerator and denominator are expressed by Laurent polynomials $\left(x_{1}-x_{2}\right) \sum_{i=0}^{3} \tilde{h}_{i}\left(x_{1}+x_{2}\right)^{i}$, where $\tilde{h}_{i}$ is depending on $f, a_{i}, b_{j}, c_{1}$ and $d_{1}$. Due to the relation $x_{1}+x_{2}=-\frac{e_{1} g}{c_{1}}$, the transformation $T_{1}(f)=\bar{f}(f, g)$ is given as a rational polynomial of bi-degree $(1,3)$ in $(f, g)$. Therefore the property (i) is proved. (ii) Eight singular points $\left(f_{s}, g_{s}\right) \in \mathbb{P}^{1} \times \mathbb{P}^{1}(s=1, \ldots, 8)$ in coordinates $(f, g)$ are on one line $g=\infty$ of bi-degree $(0,1)$ and one parabolic curve $e_{1} f^{2}+e_{1} f g+c_{1}=0$ of bi-degree $(2,1)$ as follows:

$$
\left(\frac{1}{a_{1}}, \infty\right), \quad\left(\frac{1}{b_{1}}, \infty\right), \quad\left(\frac{1}{a_{i}},-\frac{1}{a_{i}}-\frac{a_{i} c_{1}}{e_{1}}\right)_{i=2,3,4}, \quad\left(\frac{1}{b_{i}},-\frac{1}{b_{i}}-\frac{b_{i} c_{1}}{e_{1}}\right)_{i=2,3,4} .
$$

Hence the property (ii) is confirmed since the configuration (2.5) is the realization of the surface type $A_{1}^{(1)}$.

According to Remark 1.1, the system (2.2) is regarded as a variation of the $q-E_{7}^{(1)}$ system.

\footnotetext{
${ }^{11}$ The operator $T_{1}$ is generally selected as $T_{a_{i}}^{-1} T_{b_{j}}^{-1}$. The directions such as $T_{a_{i}}^{-1} T_{b_{j}}^{-1}, T_{a_{i}}^{-1} T_{a_{j}}^{-1} T_{c_{k}}^{-1}$ and $T_{a_{i}}^{-1} T_{a_{j}}^{-1} T_{d_{k}}$ are fundamental ones.
} 


\section{Lax equations}

In this section we recall Lax equations for the $q$-Garnier system [28, Section 2.1] and investigate a reduction of particular case $N=3$ of them. As a result, we obtain the Lax equations for the $q$-Painlevé type system (2.2).

\subsection{Case of the $q$-Garnier system}

The scalar Lax equations for the $q$-Garnier system are

$$
\begin{aligned}
L_{1}(x)= & A(x) F\left(\frac{x}{q}\right) y(q x)+q c_{1} c_{2} B\left(\frac{x}{q}\right) F(x) y\left(\frac{x}{q}\right) \\
& -\left\{\left(x-a_{1}\right)\left(x-b_{1}\right) F\left(\frac{x}{q}\right) G(x)+\frac{F(x)}{G\left(\frac{x}{q}\right)} V\left(\frac{x}{q}\right)\right\} y(x), \\
L_{2}(x)= & F(x) \bar{y}(x)-A_{1}(x) y(q x)+\left(x-b_{1}\right) G(x) y(x), \\
L_{3}(x)= & \bar{F}\left(\frac{x}{q}\right) y(x)+\left(x-a_{1}\right) G\left(\frac{x}{q}\right) \bar{y}(x)-q c_{1} c_{2} B_{1}\left(\frac{x}{q}\right) \bar{y}\left(\frac{x}{q}\right),
\end{aligned}
$$

where

$$
\begin{aligned}
& A(x)=\prod_{i=1}^{N+1}\left(x-a_{i}\right), \quad B(x)=\prod_{i=1}^{N+1}\left(x-b_{i}\right), \quad A_{1}(x)=\frac{A(x)}{x-a_{1}}, \quad B_{1}(x)=\frac{B(x)}{x-b_{1}}, \\
& F(x)=\sum_{i=0}^{N} f_{i} x^{i}, \quad G(x)=\sum_{i=0}^{N-1} g_{i} z^{i}, \quad V(x)=q c_{1} c_{2} A_{1}(x) B_{1}(x)-F(x) \bar{F}(x) .
\end{aligned}
$$

Here the deformation direction is $T_{1}(2.1)$ and $f_{0}, \ldots, f_{N}, g_{0}, \ldots, g_{N-1} \in \mathbb{P}^{1}$ are variables depending on parameters $a_{i}, b_{i}, c_{i}, d_{i}$ with a constraint $\prod_{i=1}^{N+1} \frac{a_{i}}{b_{i}}=q \frac{c_{1} c_{2}}{d_{1} d_{2}}$.

Remark 3.1. The scalar Lax pair $L_{1}=0$ and $L_{2}=0$ (or $L_{3}=0$ ) is equivalent to the pair of the deformation equations $L_{2}=0$ and $L_{3}=0$.

The equation $L_{1}=0$ (we call it the $L_{1}$ equation) is equivalent to one for Sakai's system given in [41] and the deformation direction is opposite to one for Sakai's system. The $q$-Garnier system is

$$
\begin{aligned}
& G(x) \underline{G}(x)=c_{1} c_{2} \frac{A_{1}(x) B_{1}(x)}{\left(x-a_{1}\right)\left(x-b_{1}\right)} \quad \text { for } \quad F(x)=0, \\
& F(x) \bar{F}(x)=q c_{1} c_{2} A_{1}(x) B_{1}(x) \quad \text { for } \quad G(x)=0, \\
& f_{N} \bar{f}_{N}=q\left(g_{N-1}-c_{1}\right)\left(g_{N-1}-c_{2}\right), \quad f_{0} \bar{f}_{0}=a_{1} b_{1}\left(g_{0}-\frac{d_{1}}{a_{1} b_{1}} A(0)\right)\left(g_{0}-\frac{d_{2}}{a_{1} b_{1}} A(0)\right),
\end{aligned}
$$

where $2 N$ variables $\frac{f_{1}}{f_{0}}, \ldots, \frac{f_{N}}{f_{0}}, g_{0}, \ldots, g_{N-1}$ are the dependent variables. Then we have the following fact ${ }^{12}$.

Proposition 3.2. The compatibility condition of the Lax pair $L_{1}=0$ and $L_{2}=0$ (3.1) is equivalent to the q-Garnier system (3.3).

\footnotetext{
${ }^{12}$ For the proof of Proposition 3.2, see [28, Section 2.3].
} 


\subsection{Reduction to the $q$-Painlevé type system}

We impose a reduction condition by a constraint of the parameters

$$
c_{1}=c_{2}, \quad d_{1}=d_{2}
$$

and specialize the dependent variables as

$$
f_{0}=f_{3}=0, \quad f_{1}=w_{1}, \quad f_{2}=-f w_{1}, \quad g_{0}=e_{1}, \quad g_{1}=e_{1} g, \quad g_{2}=c_{1},
$$

where $w_{1}^{13}$ is a "gauge freedom". Applying the conditions (3.4) and (3.5) into (3.1) and (3.3), we obtain the following linear equations

$$
\begin{aligned}
L_{1}(x)= & A(x)\left(1-\frac{f x}{q}\right) y(q x)+q^{2} c_{1}^{2} B\left(\frac{x}{q}\right)(1-f x) y\left(\frac{x}{q}\right) \\
& -\left\{\left(x-a_{1}\right)\left(x-b_{1}\right)\left(1-\frac{f x}{q}\right) \varphi(x)+\frac{q(1-f x)}{\varphi\left(\frac{x}{q}\right)} V\left(\frac{x}{q}\right)\right\} y(x), \\
L_{2}(x)= & w_{1} x(1-f x) \bar{y}(x)-A_{1}(x) y(q x)+\left(x-b_{1}\right) \varphi(x) y(x), \\
L_{3}(x)= & \bar{w}_{1} \frac{x}{q}\left(1-\bar{f} \frac{x}{q}\right) y(x)+\left(x-a_{1}\right) \varphi\left(\frac{x}{q}\right) \bar{y}(x)-q c_{1}^{2} B_{1}\left(\frac{x}{q}\right) \bar{y}\left(\frac{x}{q}\right),
\end{aligned}
$$

where $\varphi$ is given by $(2.3)$ and

$$
\begin{aligned}
& A(x)=\prod_{i=1}^{4}\left(x-a_{i}\right), \quad B(x)=\prod_{i=1}^{4}\left(x-b_{i}\right), \\
& V(x)=q c_{1}^{2} A_{1}(x) B_{1}(x)-w_{1} \bar{w}_{1} x^{2}(1-f x)(1-\bar{f} x) .
\end{aligned}
$$

Then we have

Proposition 3.3. The compatibility condition of the $L_{1}$ and $L_{2}$ equations (3.6) is equivalent to the q-Painlevé type system (2.2).

Proof. Thanks to Proposition 3.2 and the conditions (3.4), (3.5).

The pair of $L_{1}$ and $L_{2}$ equations (3.6) is regard as the Lax pair for the system (2.2).

\section{Characterization of the $L_{1}$ equation}

In [48] Y. Yamada has formulated a Lax form for the $q$-Painlevé equation of type $E_{7}^{(1)}$ as the linear equation (say $L_{1}=0$ ) and its deformed equation. Our direction $T_{1}(2.1)$ is different from Yamada's one. In general the $L_{1}$ equation is expressed in terms of different dependent variables according to several deformation directions. In this section, from the viewpoint of coordinates of dependent variables, we study a connection between our $L_{1}$ equation (3.6) and Yamada's $L_{1}$ equation.

\footnotetext{
${ }^{13}$ For convenience, $f_{1}$ is replaced by a different symbol $w_{1}$ since $f_{1}$ looks like $f$.
} 


\subsection{Case of our $L_{1}$ equation}

We consider characterizing our $L_{1}$ equation (3.6) in the coordinates $(f, g) \in \mathbb{P}^{1} \times \mathbb{P}^{1}$. The compatibility of $L_{2}$ and $L_{3}$ equations (3.6) gives the first equation of (2.2) and two relations

$$
\begin{aligned}
& w_{1} \bar{w}_{1} x_{1}^{2}\left(1-f x_{1}\right)\left(1-\bar{f} x_{1}\right)=q c_{1}^{2} A_{1}\left(x_{1}\right) B_{1}\left(x_{1}\right), \\
& w_{1} \bar{w}_{1} x_{2}^{2}\left(1-f x_{2}\right)\left(1-\bar{f} x_{2}\right)=q c_{1}^{2} A_{1}\left(x_{2}\right) B_{1}\left(x_{2}\right) .
\end{aligned}
$$

The relations (4.1) and (4.2) give the second equation of (2.2). Eliminating $\bar{f}, w_{1}$ and $\bar{w}_{1}$ from the expression $L_{1}$ (3.6) by using the first equation of (2.2) and the relation (4.1), the expression $L_{1}$ (3.6) is rewritten in terms of variables $f$ and $x_{1}$ as the expression

$$
\begin{aligned}
L_{1}(x)= & A(x)\left(1-f \frac{x}{q}\right)\left[y(q x)-\frac{\left(x-b_{1}\right) \varphi(x)}{A_{1}(x)} y(x)\right] \\
& +q^{2} c_{1}^{2} B\left(\frac{x}{q}\right)(1-f x)\left[y\left(\frac{x}{q}\right)-\frac{A_{1}\left(\frac{x}{q}\right)}{\left(\frac{x}{q}-b_{1}\right) \varphi\left(\frac{x}{q}\right)}\right] \\
& +\frac{c_{1}^{2} x^{2}(1-f x)\left(1-f \frac{x}{q}\right)}{\left(x_{1}-x_{2}\right) \varphi\left(\frac{x}{q}\right)}\left[\frac{\left(\frac{x}{q}-x_{2}\right) A_{1}\left(x_{1}\right) B_{1}\left(x_{1}\right)}{x_{1}^{2}\left(1-f x_{1}\right)}-\frac{\left(\frac{x}{q}-x_{1}\right) A_{1}\left(x_{2}\right) B_{1}\left(x_{2}\right)}{x_{2}^{2}\left(1-f x_{2}\right)}\right] y(x),
\end{aligned}
$$

where $x_{2}=\frac{e_{1}}{c_{1} x_{1}}$. Next when we set the expression $L_{1}^{*}$ by

$$
L_{1}^{*}(x)=\left(1-f x_{1}\right)\left(1-f x_{2}\right) L_{1}(x) .
$$

Then we have:

Proposition 4.1. The $L_{1}^{*}$ equation (4.4) has the following characterization ${ }^{14}$ :

(i) The expression $L_{1}^{*}(f, g)$ is a polynomial of bi-degree $(3,2)$ in the coordinates $(f, g) \in \mathbb{P}^{1} \times \mathbb{P}^{1}$.

(ii) As a polynomial, the expression $L_{1}^{*}(f, g)$ vanishes at the following 12 points $\left(f_{s}, g_{s}\right) \in$ $\mathbb{P}^{1} \times \mathbb{P}^{1}(s=1, \ldots, 12):$

$$
\begin{aligned}
& \left(\frac{1}{a_{1}}, \infty\right), \quad\left(\frac{1}{b_{1}}, \infty\right), \quad\left(\frac{1}{a_{i}},-\frac{1}{a_{i}}-\frac{a_{i} c_{1}}{e_{1}}\right)_{i=2,3,4}, \quad\left(\frac{1}{b_{i}},-\frac{1}{b_{i}}-\frac{b_{i} c_{1}}{e_{1}}\right)_{i=2,3,4}, \\
& \left(\frac{q}{x}, \infty\right), \quad\left(\frac{1}{x},-\frac{1}{x}-\frac{c_{1} x}{e_{1}}\right), \quad\left(\frac{1}{x}, g_{\frac{1}{x}}\right), \quad\left(\frac{q}{x}, g_{\frac{q}{x}}\right),
\end{aligned}
$$

where the first 8 points are as in (2.5) and $g_{u}$ is given by

$$
\frac{y\left(\frac{q}{u}\right)}{y\left(\frac{1}{u}\right)}=: \frac{\left(\frac{1}{u}-b_{1}\right)\left(e_{1}+\frac{e_{1} g_{u}}{u}+\frac{c_{1}}{u^{2}}\right)}{A_{1}\left(\frac{1}{u}\right)}, \quad u=\frac{1}{x}, \frac{q}{x} .
$$

Conversely the $L_{1}^{*}$ equation is uniquely characterized by these properties $(i)$ and $(i i)$.

Proof. By the expression $L_{1}(4.3)$, the expression $L_{1}^{*}$ is rewritten in terms of variables $f$ and $x_{1}$ as follows

$$
\begin{aligned}
L_{1}^{*}(x)= & A(x)\left(1-f x_{1}\right)\left(1-f x_{2}\right)\left(1-f \frac{x}{q}\right) y(q x) \\
& +q^{2} c_{1}^{2}\left(1-f x_{1}\right)\left(1-f x_{2}\right)(1-f x) y\left(\frac{x}{q}\right)+P(x) y(x),
\end{aligned}
$$

\footnotetext{
${ }^{14}$ For other cases, see $[16,46]\left(\right.$ case $\left.e-E_{8}^{(1)}\right),[16,48]\left(\right.$ case $\left.q-E_{8}^{(1)}\right)$.
} 
where

$$
\begin{aligned}
P(x)= & \frac{q^{2} c_{1}^{2}(1-f x)\left(1-f \frac{x}{q}\right)\left(\frac{x}{q}\right)^{2}}{\left(x_{1}-x_{2}\right) \varphi\left(\frac{x}{q}\right)} Q(x) \\
& -\left(x-a_{1}\right)\left(x-b_{1}\right)\left(1-f x_{1}\right)\left(1-f x_{2}\right)\left(1-f \frac{x}{q}\right) \varphi(x), \\
Q(x)= & \frac{\left(1-f x_{2}\right)\left(\frac{x}{q}-x_{2}\right) A_{1}\left(x_{1}\right) B_{1}\left(x_{1}\right)}{x_{1}^{2}}-\frac{\left(1-f x_{1}\right)\left(\frac{x}{q}-x_{1}\right) A_{1}\left(x_{2}\right) B_{1}\left(x_{2}\right)}{x_{2}^{2}} \\
& -\frac{\left(x_{1}-x_{2}\right)\left(1-f x_{1}\right)\left(1-f x_{2}\right) A_{1}\left(\frac{x}{q}\right) B_{1}\left(\frac{x}{q}\right)}{\left(1-f \frac{x}{q}\right)\left(\frac{x}{q}\right)^{2}},
\end{aligned}
$$

and $x_{1}, x_{2}\left(=\frac{e_{1}}{c_{1} x_{1}}\right)$ are as in Section 3.2. Similarly to the proof of Proposition 2.1, the expression $Q(x)$ is given as a Laurent polynomial $\left(x_{1}-x_{2}\right) \sum_{i=0}^{3} k_{i}\left(x_{1}+x_{2}\right)^{i}$ where $k_{i}$ depends on $f$, $a_{i}, b_{i}, c_{1}$ and $d_{1}$. The expression $Q(x)$ has zeros at $x=q x_{1}, q x_{2}$ which are solutions of the equation $\varphi\left(\frac{x}{q}\right)=0$. Therefore, due to the relation $x_{1}+x_{2}=-\frac{e_{1} g}{c_{1}}$, the expression $P(x)$ (i.e., the coefficient of $y(x))$ is a polynomial of bi-degree $(3,2)$ in $(f, g)$. It is obvious that the coefficients of $y(q x)$ and $y\left(\frac{x}{q}\right)$ are polynomials of bi-degree $(3,1)$ in $(f, g)$. Hence the property (i) is completely proved. Next the property (ii) can be easily confirmed by substituting the 12 points (4.5) into the expression $L_{1}^{*}(4.6)$.

\subsection{Case of Yamada's $L_{1}$ equation}

Firstly we recall the well-known $q-E_{7}^{(1)}$ system [5] and the corresponding Yamada's Lax form [48]. Next we characterize Yamada's $L_{1}$ equation in terms of dependent variables. The complex parameters $a_{i}, b_{i}, c_{1}$ and $d_{1}$ be as in Section 2 and let $(\lambda, \mu) \in \mathbb{P}^{1} \times \mathbb{P}^{1}$ be dependent variables. Then we consider a $q$-shift operator $T_{2}{ }^{15}$ as

$$
T_{2}=T_{a_{1}}^{-1} T_{a_{2}}^{-1} T_{b_{1}}^{-1} T_{b_{2}}^{-1}
$$

The operator $T_{2}$ plays the role of the evolution of the $q-E_{7}^{(1)}$ system. The system is well-known as the bi-rational transformation $T_{2}^{-1}(\mu)=\underline{\mu}(\lambda, \mu)$ and $T_{2}(\lambda)=\bar{\lambda}(\lambda, \mu)$ in $\mathbb{P}^{1} \times \mathbb{P}^{1}$ as follows $[5,13,16,39]$

$$
\begin{aligned}
& \frac{\left(\lambda \mu-\frac{e_{2}}{c_{1}}\right)\left(\lambda \underline{\mu}-\frac{e_{2}}{q c_{1}}\right)}{(\lambda \mu-1)(\lambda \underline{\mu}-1)}=\frac{e_{2}^{2}}{q c_{1}^{2}} \frac{\prod_{i=1,2}\left(1-a_{i} \lambda\right)\left(1-b_{i} \lambda\right)}{\prod_{i=3,4}\left(1-a_{i} \lambda\right)\left(1-b_{i} \lambda\right)}, \\
& \frac{\left(\lambda \mu-\frac{e_{2}}{c_{1}}\right)\left(\bar{\lambda} \mu-\frac{q e_{2}}{c_{1}}\right)}{(\lambda \mu-1)(\bar{\lambda} \mu-1)}=\frac{\prod_{i=1,2}\left(\mu-\frac{a_{i} e_{2}}{c_{1}}\right)\left(\mu-\frac{b_{i} e_{2}}{c_{1}}\right)}{\prod_{i=3,4}\left(\mu-a_{i}\right)\left(\mu-b_{i}\right)},
\end{aligned}
$$

where $e_{2}=\frac{a_{3} a_{4} d_{1}}{b_{1} b_{2}}$. Here eight singular points $\left(\lambda_{s}, \mu_{s}\right)(s=1, \ldots, 8)$ in coordinates $(\lambda, \mu)$ are on two curves $\lambda \mu=1$ and $\lambda \mu=\frac{e_{2}}{c_{1}}$ as follows

$$
\left(\frac{1}{a_{i}}, \frac{a_{i} e_{2}}{c_{1}}\right)_{i=1,2}, \quad\left(\frac{1}{b_{i}}, \frac{b_{i} e_{2}}{c_{1}}\right)_{i=1,2}, \quad\left(\frac{1}{a_{i}}, a_{i}\right)_{i=3,4}, \quad\left(\frac{1}{b_{i}}, b_{i}\right)_{i=3,4} .
$$

\footnotetext{
${ }^{15}$ The direction $T_{2}$ is given by a composition of the fundamental ones such as $T_{1}(2.1)$ and $T_{a_{2}}^{-1} T_{b_{2}}^{-1}$.
} 
The following scalar Lax equations

$$
\begin{aligned}
L_{1}(x)= & \frac{A(x)}{1-\lambda x}\left[y(q x)-\frac{e_{2} \prod_{i=1,2}\left(x-b_{i}\right)(\mu-x)}{\prod_{i=3,4}\left(x-a_{i}\right)\left(\mu-\frac{e_{2} x}{c_{1}}\right)} y(x)\right] \\
& +\frac{q^{2} c_{1}^{2} B\left(\frac{x}{q}\right)}{1-\lambda \frac{x}{q}}\left[y\left(\frac{x}{q}\right)-\frac{\prod_{i=3,4}\left(\frac{x}{q}-a_{i}\right)\left(\mu-\frac{e_{2} x}{q c_{1}}\right)}{e_{2} \prod_{i=1,2}\left(\frac{x}{q}-b_{i}\right)\left(\mu-\frac{x}{q}\right)} y(x)\right] \\
& +\frac{c_{1}\left(1-\frac{c_{1}}{e_{2}}\right) x^{2}}{\mu}\left[\frac{\prod_{i=3,4}\left(\mu-a_{i}\right)\left(\mu-b_{i}\right)}{(\lambda \mu-1)\left(\mu-\frac{x}{q}\right)}-\frac{\prod_{i=1,2}\left(\mu-\frac{a_{i} e_{2}}{c_{1}}\right)\left(\mu-\frac{b_{i} e_{2}}{c_{1}}\right)}{\left(\lambda \mu-\frac{e_{2}}{c_{1}}\right)\left(\mu-\frac{e_{2} x}{c_{1}}\right)}\right] y(x), \quad(4 . \\
L_{2}(x)= & w_{2} x(1-\lambda x) \bar{y}(x)-\prod_{i=3}^{4}\left(x-a_{i}\right)\left(1-\frac{e_{2} x}{c_{1} \mu}\right) y(q x)+e_{2} \prod_{i=1}^{2}\left(x-b_{i}\right)\left(1-\frac{x}{\mu}\right) y(z)
\end{aligned}
$$

are equivalent to those in $[16,24,48]$ up to a gauge transformation of $y(x)$. Here $A$ and $B$ are as in (3.7) and $w_{2}$ is a gauge freedom (as mentioned in Section 3.2). Then the compatibility of the $L_{1}$ and $L_{2}$ equations (4.10) is equivalent to the system (4.8). Next setting the expression $L_{1}^{*}$ by

$$
L_{1}^{*}(x)=(1-\lambda x)\left(1-\lambda \frac{x}{q}\right)(\lambda \mu-1)\left(\lambda \mu-\frac{e_{2}}{c_{1}}\right) L_{1}(x),
$$

we have:

Proposition 4.2. The $L_{1}^{*}$ equation (4.11) has the following characterization:

(i) The expression $L_{1}^{*}$ is a polynomial of bi-degree $(3,2)$ in the coordinates $(\lambda, \mu) \in \mathbb{P}^{1} \times \mathbb{P}^{1}$.

(ii) As a polynomial, the expression $L_{1}^{*}$ vanishes at the following 12 points $\left(\lambda_{s}, \mu_{s}\right) \in \mathbb{P}^{1} \times \mathbb{P}^{1}$ $(s=1, \ldots, 12)$ :

$$
\begin{aligned}
& \left(\frac{1}{a_{i}}, \frac{a_{i} e_{2}}{c_{1}}\right)_{i=1,2}, \quad\left(\frac{1}{b_{i}}, \frac{b_{i} e_{2}}{c_{1}}\right)_{i=1,2}, \quad\left(\frac{1}{a_{i}}, a_{i}\right)_{i=3,4}, \quad\left(\frac{1}{b_{i}}, b_{i}\right)_{i=3,4}, \\
& \left(\frac{1}{x}, x\right), \quad\left(\frac{q}{x}, \frac{e_{2} x}{q c_{1}}\right), \quad\left(\frac{1}{x}, \mu_{\frac{1}{x}}\right), \quad\left(\frac{q}{x}, \mu_{\frac{q}{x}}\right),
\end{aligned}
$$

where the first 8 points are as in (4.9) and $\mu_{u}$ is given by

$$
\frac{Y\left(\frac{q}{u}\right)}{Y\left(\frac{1}{u}\right)}=: \frac{e_{2} \prod_{i=1,2}\left(\frac{1}{u}-b_{i}\right)\left(\mu_{u}-\frac{1}{u}\right)}{\prod_{i=3,4}\left(\frac{1}{u}-a_{i}\right)\left(\mu_{u}-\frac{e_{2}}{c_{1} u}\right)}, \quad u=\frac{1}{x}, \frac{q}{x} .
$$

Conversely the equation $L_{1}^{*}$ is uniquely characterized by these properties $(i)$ and $(i i)$.

Proof. This proof is the similar as for Proposition 4.1.

\subsection{Correspondence between two $L_{1}$ equations}

Thanks to Propositions 4.1 and 4.2, we have: 
Theorem 4.3. The $L_{1}$ equation (4.3) is equivalent to Yamada's $L_{1}$ equation (4.10) under a relation

$$
f=\lambda, \quad \frac{\left(\lambda \mu-\frac{e_{2}}{c_{1}}\right)\left(e_{1} f^{2}+e_{1} f g+c_{1}\right)}{e_{2}\left(1-b_{2} \lambda\right)(\lambda \mu-1)\left(1-a_{2} f\right)}=1 .
$$

Proof. Comparing the last two points of (4.5) in Proposition 4.1 with ones of (4.12) in Proposition 4.2 , we obtain the transformation (4.13) as a necessary condition to change the $L_{1}^{*}$ equation (4.11) into the $L_{1}^{*}$ equation (4.4). Conversely, under the relation (4.13), $\mu(f, g)$ is written as a rational function with the numerator and the denominator of bi-degree $(1,1)$ in $(f, g)$ respectively. Substituting the expression $\mu(f, g)$ (4.13) into the $L_{1}^{*}$ equation (4.11), it is shown that the algebraic curve $L_{1}^{*}=0$ (4.11) of bi-degree $(3,2)$ in $(\lambda, \mu)$ changes to the algebraic curve of bi-degree $(5,2)$ in $(f, g)$. Furthermore, due to the relation (4.13), 12 points (4.12) are changed to 12 points (4.5) and 2 lines $f=\frac{1}{a_{2}}, f=\frac{1}{b_{2}}$. Namely it turns out that the algebraic curve $L_{1}^{*}(\lambda, \mu)=0$ (4.11) of bi-degree $(3,2)$ is changed to the algebraic curve $L_{1}^{*}(f, g) \times\left(1-a_{2} f\right)\left(1-b_{2} f\right)=0(4.4)$ of bi-degree $(3,2) \times(2,0)$ in $(f, g)$. Hence, the relation (4.13) is proved to be the sufficient condition that the $L_{1}$ equation (4.10) corresponds with the $L_{1}$ equation (4.3).

We note that the system (2.2) has the Lax pair whose $L_{1}$ equation (4.3) is equivalent to that (4.10) of the $q-E_{7}^{(1)}$ system (4.8) and clarify the relation (4.13) of the dependent variables between the systems (2.2) and (4.8).

\section{Particular solutions}

In this section we recall the particular solutions for the system (3.3) given in [28], and derive ones for the system (2.2) by the similar reduction as in Section 3.2.

\subsection{Case of the $q$-Garnier system}

The contents are extracts from [28, Section 5.2]. For convenience we change the notations in Section 3.1 as follows

$$
\left[\begin{array}{cccc}
a_{1} & \ldots & a_{N} & a_{N+1} \\
b_{1} & \ldots & b_{N} & b_{N+1} \\
c_{1} & c_{2} & d_{1} & d_{2}
\end{array}\right] \mapsto\left[\begin{array}{cccc}
\frac{1}{a_{1}} & \ldots & \frac{1}{a_{N}} & q^{m+n} \\
\frac{1}{b_{1}} & \ldots & \frac{1}{b_{N}} & \frac{1}{q} \\
c q^{n} \prod_{1}^{N} \frac{b_{i}}{a_{i}} & q^{m} & c & 1
\end{array}\right]
$$

where $a_{1}, \ldots, a_{N}, b_{1}, \ldots, b_{N}, c \in \mathbb{C}^{\times}$and $m, n \in \mathbb{Z}_{\geq 0}$. Correspondingly we replace the notations $A, B$ etc. (3.2) by

$$
A(x)=\prod_{i=1}^{N}\left(a_{i} x\right)_{1}, \quad B(x)=\prod_{i=1}^{N}\left(b_{i} x\right)_{1}, \quad A_{1}(x)=\frac{A(x)}{\left(a_{1} x\right)_{1}}, \quad B_{1}(x)=\frac{B(x)}{\left(b_{1} x\right)_{1}} .
$$

We also replace the evolution direction (2.1) by

$$
T_{1}=T_{a_{1}} T_{b_{1}} .
$$

We show particular solutions in terms of the $\tau$ function

$$
\tau_{m, n}=\operatorname{det}\left[N+1 \varphi_{N}\left(\begin{array}{c}
b_{1}, \ldots, b_{N}, q^{-(m+n)} \\
a_{1}, \ldots, a_{N}
\end{array}, c q^{i+j+1}\right)\right]_{i, j=0}^{n},
$$


where the generalized $q$-hypergeometric function ${ }_{N+1} \varphi_{N}{ }^{16}[3]$ is defined by (1.1). Then we have the following fact ${ }^{17}$.

Proposition 5.1. The polynomials $F(x)$ and $G(x)$ determined by

$$
\begin{aligned}
& \frac{F\left(\frac{1}{a_{i}}\right)}{F\left(\frac{1}{b_{j}}\right)}=\alpha \frac{T_{a_{i}}\left(\tau_{m, n}\right) T_{a_{i}}^{-1}\left(\tau_{m+1, n-1}\right)}{T_{b_{j}}^{-1}\left(\tau_{m, n}\right) T_{b_{j}}\left(\tau_{m+1, n-1}\right)}, \quad i, j=1, \ldots, N, \\
& G\left(\frac{1}{a_{i}}\right)=\beta \frac{T_{a_{i}}\left(\bar{\tau}_{m, n}\right) T_{a_{i}}^{-1}\left(\tau_{m+1, n-1}\right)}{T_{a_{1}}\left(\tau_{m, n}\right) T_{a_{1}}^{-1}\left(\bar{\tau}_{m+1, n-1}\right)}, \quad i=2, \ldots, N, \\
& G\left(\frac{1}{b_{i}}\right)=\gamma \frac{T_{b_{i}}^{-1}\left(\tau_{m, n}\right) T_{b_{i}}\left(\bar{\tau}_{m+1, n-1}\right)}{T_{b_{1}}^{-1}\left(\bar{\tau}_{m, n}\right) T_{b_{1}}\left(\tau_{m+1, n-1}\right)}, \quad i=2, \ldots, N,
\end{aligned}
$$

give particular solutions of a bi-rational equation

$$
\begin{aligned}
& G(x) \underline{G}(x)=\frac{c\left(q x, \frac{x}{q^{m+n}}\right)_{1} A_{1}(x) B_{1}(x)}{\left(a_{1} x, b_{1} x\right)_{1}} \quad \text { for } \quad F(x)=0 \\
& F(x) \bar{F}(x)=\left(q x, \frac{x}{q^{m+n}}\right)_{1} A_{1}(x) B_{1}(x) \quad \text { for } \quad G(x)=0 \\
& f_{N} \bar{f}_{N}=\frac{q a_{1} b_{1}}{c}\left(g_{N-1}-\frac{c \prod_{i=2}^{N}\left(-b_{i}\right)}{a_{1} q^{m-1}}\right)\left(g_{N-1}-\frac{\prod_{i=2}^{N}\left(-a_{i}\right)}{b_{1} q^{n}}\right), \quad f_{0} \bar{f}_{0}=\left(g_{0}, \frac{g_{0}}{c}\right)_{1} .
\end{aligned}
$$

Here $\alpha, \beta$ and $\gamma$ are given by

$$
\begin{aligned}
& \alpha=-c q^{n-m} \frac{\left(a_{i} q^{m+n}\right)_{1}\left(\frac{a_{i}}{q}\right)_{1}^{n}\left(\frac{b_{j}}{q}\right)_{1}^{n}}{\left(a_{i}\right)_{1}^{n+1}\left(b_{j}\right)_{1}^{n}} \frac{B\left(\frac{1}{a_{i}}\right)}{A\left(\frac{1}{b_{j}}\right)}, \\
& \beta=c \frac{\left(b_{1}, a_{i} q^{m+n}\right)_{1}\left(\frac{a_{i}}{q}\right)_{1}^{n} B_{1}\left(\frac{1}{a_{i}}\right)}{a_{1} q^{m}\left(\frac{b_{1}}{a_{1}}\right)_{1}\left(a_{i}\right)_{1}^{n+1}}, \quad \gamma=\frac{\left(a_{1}\right)_{1}\left(b_{i}\right)_{1}^{n} A_{1}\left(\frac{1}{b_{i}}\right)}{b_{1} q^{n}\left(\frac{a_{1}}{b_{1}}\right)_{1}\left(\frac{b_{i}}{q}\right)_{1}^{n}} .
\end{aligned}
$$

\subsection{Reduction to the $q$-Painlevé type system}

In this subsection we derive particular solutions for the Painlevé type equation (2.2). In a similar way as in Section 3.2, we consider the reduction from the particular case $N=3$ of the $q$-Garnier system (3.3). In order to do this, we impose a constraint of parameters

$$
c=1, \quad q^{m-n} \prod_{i=1}^{3} \frac{a_{i}}{b_{i}}=1,
$$

and the specialization (3.5). Then the tau function $\tau_{m, n}(5.4)$ is reduced to the following function

$$
\tau_{m, n}=\operatorname{det}\left[{ }_{4} \varphi_{3}\left(\begin{array}{c}
b_{1}, b_{2}, b_{3}, q^{-(m+n)} \\
a_{1}, a_{2}, a_{3}
\end{array}, q^{i+j+1}\right)\right]_{i, j=0}^{n},
$$

where the generalized $q$-hypergeometric function ${ }_{4} \varphi_{3}$ is defined by (1.1). As the case of a reduction of Proposition 5.1, we have the following ${ }^{18}$.

\footnotetext{
${ }^{16}$ In $[43,44]$ the particular solutions of a higher order $q$-Painlevé system was constructed in terms of the $q$-hypergeometric function ${ }_{N+1} \varphi_{N}$ by T. Suzuki.

${ }^{17}$ For the proof of Proposition 5.1, see [28, Section 5].

${ }^{18}$ As another derivation of the particular solutions (5.7), see Section A.2 (Padé interpolation method).
} 
Proposition 5.2. The particular values of $f$ and $g$ determined by

$$
\begin{aligned}
& \frac{1-\frac{f}{a_{i}}}{1-\frac{f}{b_{j}}}=\alpha^{\prime} \frac{T_{a_{i}}\left(\tau_{m, n}\right) T_{a_{i}}^{-1}\left(\tau_{m+1, n-1}\right)}{T_{b_{j}}^{-1}\left(\tau_{m, n}\right) T_{b_{j}}\left(\tau_{m+1, n-1}\right)}, \quad i, j=1,2,3, \\
& 1+\frac{g}{a_{i}}+\frac{\kappa}{a_{i}^{2}}=\beta^{\prime} \frac{T_{a_{i}}\left(\bar{\tau}_{m, n}\right) T_{a_{i}}^{-1}\left(\tau_{m+1, n-1}\right)}{T_{a_{1}}\left(\tau_{m, n}\right) T_{a_{1}}^{-1}\left(\bar{\tau}_{m+1, n-1}\right)}, \quad i=2,3, \\
& 1+\frac{g}{b_{i}}+\frac{\kappa}{b_{i}^{2}}=\gamma^{\prime} \frac{T_{b_{i}}^{-1}\left(\tau_{m, n}\right) T_{b_{i}}\left(\bar{\tau}_{m+1, n-1}\right)}{T_{b_{1}}^{-1}\left(\bar{\tau}_{m, n}\right) T_{b_{1}}\left(\tau_{m+1, n-1}\right)}, \quad i=2,3,
\end{aligned}
$$

give particular solutions of the following overdetermined bi-rational equation:

$$
\begin{aligned}
& \left(f^{2}+g f+\kappa\right)\left(f^{2}+\underline{g} f+q \kappa\right)=\frac{\left(f-\frac{1}{q^{m+n}}\right)(f-q) \prod_{i=2,3}\left(f-a_{i}\right)\left(f-b_{i}\right)}{\left(f-a_{1}\right)\left(f-b_{1}\right)}, \\
& \frac{x_{1}^{2}\left(1-f x_{1}\right)\left(1-\bar{f} x_{1}\right)}{x_{2}^{2}\left(1-f x_{2}\right)\left(1-\bar{f} x_{2}\right)}=\frac{\left(1-\frac{x_{1}}{q^{m+n}}\right)\left(1-q x_{1}\right) \prod_{i=2,3}\left(1-a_{i} x_{1}\right)\left(1-b_{i} x_{1}\right)}{\left(1-\frac{x_{2}}{q^{m+n}}\right)\left(1-q x_{2}\right) \prod_{i=2,3}\left(1-a_{i} x_{2}\right)\left(1-b_{i} x_{2}\right)} .
\end{aligned}
$$

Here $\alpha^{\prime}, \beta^{\prime}$ and $\gamma^{\prime}$ are given by

$$
\begin{aligned}
& \alpha^{\prime}=-q^{n-m} \frac{a_{i}\left(a_{i} q^{m+n}\right)_{1}\left(\frac{a_{i}}{q}, \frac{b_{j}}{q}\right)_{1}^{n}}{b_{j}\left(a_{i}\right)_{1}^{n+1}\left(b_{j}\right)_{1}^{n}} \frac{\prod_{s=1}^{3}\left(\frac{b_{s}}{a_{i}}\right)_{1}}{\prod_{s=1}^{3}\left(\frac{a_{s}}{b_{j}}\right)_{1}}, \\
& \beta^{\prime}=\frac{\left(b_{1}, a_{i} q^{m+n}, \frac{b_{2}}{a_{i}}, \frac{b_{3}}{a_{i}}\right)_{1}\left(\frac{a_{i}}{q}\right)_{1}^{n}}{a_{1} q^{m}\left(\frac{b_{1}}{a_{1}}\right)_{1}\left(a_{i}\right)_{1}^{n+1}}, \quad \gamma^{\prime}=\frac{\left(a_{1}, \frac{a_{2}}{b_{i}}, \frac{a_{3}}{b_{i}}\right)_{1}\left(b_{i}\right)_{1}^{n}}{b_{1} q^{n}\left(\frac{a_{1}}{b_{1}}\right)_{1}\left(\frac{b_{2}}{q}\right)_{1}^{n}},
\end{aligned}
$$

and the evolution direction is as in $T_{1}$ (5.3) and $x=x_{1}, x_{2}$ are solutions of an equation $\varphi=0$ :

$$
\varphi(x)=1+g x+\kappa x^{2},
$$

where $\kappa=\frac{a_{2} a_{3}}{b_{1} q^{n}}$.

Proof. Substituting the conditions (5.6) and (3.5) into the particular solutions (5.5), we obtain (5.7).

\section{Conclusions}

The main results of this paper are the following.

- We showed in Proposition 2.1 that the q-Painlevé type system (2.2) is the bi-rational transformation and is related to the novel realization (i.e., configuration) (2.5) of the symmetry/surface of type $E_{7}^{(1)} / A_{1}^{(1)}$. Then the system $(2.2)$ turned out to be a variation of the $q-E_{7}^{(1)}$ system (4.8).

- We obtained the Lax equations (3.6) for the system (2.2) from the reduction of the particular case $N=3$ of the $q$-Garnier system (3.3), and clarified the connection between the system (2.2) and the $q-E_{7}^{(1)}$ system (4.8) by comparing their $L_{1}$ equations in Theorem 4.3.

- In Proposition 5.2 the determinant formulas of the particular solutions for the system (2.2) was expressed in terms of the generalized $q$-hypergeometric function ${ }_{4} \varphi_{3}$ through the similar reduction of the particular case $N=3$. 
Extending the results of this paper, we naturally have the following open problems. One may consider several variations of the $q-E_{7}$ system according to several deformation direction such as $T_{1}$ and $T_{2}$, and investigates a connection among these systems. We will carry out similar research on discrete Painlevé and Garnier systems [29]. It seems to be interesting to study reductions of cases $N \geq 4$ of the $q$-Garnier system.

\section{A From Padé interpolation to $q$-Painlevé type system}

By using a Padé interpolation problem with q-grid, in [24] we derived the scalar Lax pair, the evolution equation and the particular solutions for the $q-E_{7}^{(1)}$ system. In this appendix, in a similar manner as in [24], we directly derive the data of the $q$-Painlevé type system (2.2) given in Sections 3.2 and 5.2.

\section{A.1 Scalar Lax pair and evolution equation}

Suppose we have complex parameters $q(|q|<1), a_{1}, a_{2}, a_{3}, b_{1}, b_{2}$ and $b_{3} \in \mathbb{C}^{\times}$with the constraint (5.6). Then we consider a function

$$
\psi(x)=\prod_{i=1}^{3} \frac{\left(a_{i} x, b_{i}\right)_{\infty}}{\left(a_{i}, b_{i} x\right)_{\infty}} .
$$

Let $P(x)$ and $Q(x)$ be polynomials of degree $m$ and $n \in \mathbb{Z}_{\geq 0}$ in $x$. Then we assume that the polynomials $P$ and $Q$ satisfy the following Padé interpolation condition:

$$
\psi\left(x_{s}\right)=\frac{P\left(x_{s}\right)}{Q\left(x_{s}\right)}, \quad x_{s}=q^{s}, \quad s=0,1, \ldots, m+n .
$$

The common normalizations of the polynomials $P$ and $Q$ in $x$ are fixed as $P(0)=1$. The parameter shift operator is given by $T_{1}$ (5.3). Consider two linear relations: $L_{2}=0$ among $y(x)$, $y(q x), \bar{y}(x)$ and $L_{3}=0$ among $y(x), \bar{y}(x), \bar{y}\left(\frac{x}{q}\right)$ satisfied by the functions $y=P$ and $y=\psi Q$. Then we have:

Proposition A.1. The linear relations $L_{2}$ and $L_{3}{ }^{19}$ can be expressed as follows

$$
\begin{aligned}
& L_{2}(x)=C_{0} x(f x)_{1} \bar{y}(x)-\left(\frac{x}{q^{m+n}}\right)_{1} A_{1}(x) y(q x)+\left(b_{1} x\right)_{1} \varphi(x) y(x)=0, \\
& L_{3}(z)=C_{1} \frac{x}{q}\left(\frac{\bar{f} x}{q}\right)_{1} y(x)+\left(a_{1} x\right)_{1} \varphi\left(\frac{x}{q}\right) \bar{y}(x)-(x)_{1} B_{1}\left(\frac{x}{q}\right) \bar{y}\left(\frac{x}{q}\right)=0,
\end{aligned}
$$

where $\varphi$ is given by (5.9) and $A_{1}, B_{1}$ are the same as the case $N=3$ of (5.2). Here $f, g, C_{0}, C_{1} \in$ $\mathbb{P}^{1}$ are constants depending on parameters $a_{i}, b_{j} \in \mathbb{C}^{\times}, m, n \in \mathbb{Z}_{\geq 0}$.

Proof. By the definition of the relations $L_{2}=0$ and $L_{3}=0$, they can be written as

$$
\begin{aligned}
& L_{2}(x) \propto\left|\begin{array}{lll}
y(x) & y(q x) & \bar{y}(x) \\
\mathbf{y}(x) & \mathbf{y}(q x) & \overline{\mathbf{y}}(x)
\end{array}\right|=D_{1}(x) \bar{y}(x)-D_{2}(x) y(q x)+D_{3}(x) y(x)=0, \\
& L_{3}(x) \propto\left|\begin{array}{lll}
y(x) & \bar{y}(x) & \bar{y}\left(\frac{x}{q}\right) \\
\mathbf{y}(x) & \overline{\mathbf{y}}(x) & \overline{\mathbf{y}}\left(\frac{x}{q}\right)
\end{array}\right|=\bar{D}_{1}\left(\frac{x}{q}\right) y(x)+D_{3}\left(\frac{x}{q}\right) \bar{y}(x)-D_{2}(x) \bar{y}\left(\frac{x}{q}\right)=0,
\end{aligned}
$$

\footnotetext{
${ }^{19} L_{2}=0$ and $L_{3}=0$ (A.3) can be derived by substituting (5.1), (5.6) and $w_{1} \rightarrow-\frac{q^{2 m} C_{0}}{a_{2} a_{3}}, \bar{w}_{1} \rightarrow-\frac{q^{2 m} C_{1}}{b_{2} b_{3}}$ into $L_{2}=0$ and $L_{3}=0(3.6)$.
} 
where $\mathbf{y}(x)=\left[\begin{array}{c}P(x) \\ \psi(x) Q(x)\end{array}\right]$ and Casorati determinants

$$
D_{1}(x)=|\mathbf{y}(x), \mathbf{y}(q x)|, \quad D_{2}(x)=|\mathbf{y}(x), \overline{\mathbf{y}}(x)|, \quad D_{3}(x)=|\mathbf{y}(q x), \overline{\mathbf{y}}(x)| .
$$

Taking note of the relations

$$
\frac{\psi(q x)}{\psi(x)}=\frac{B(x)}{A(x)}, \quad \frac{\bar{\psi}(x)}{\psi(x)}=\frac{\left(a_{1}, b_{1} x\right)_{1}}{\left(a_{1} x, b_{1}\right)_{1}}
$$

where $A$ and $B$ are the same as the case $N=3$ of (5.2), we rewrite the Casorati determinants (A.5) into the following determinants

$$
\begin{aligned}
& D_{1}(x)=\frac{\psi(x)}{A(x)} R_{1}(x)=: \frac{\psi(x)}{A(x)} \prod_{i=0}^{m+n-1}\left(\frac{x}{q^{i}}\right)_{1} c_{0} x(f x)_{1}, \\
& D_{2}(x)=\frac{\psi(x)}{\left(a_{1} x, b_{1}\right)_{1}} R_{2}(x)=: \frac{\psi(x)}{\left(a_{1} x, b_{1}\right)_{1}} \prod_{i=0}^{m+n}\left(\frac{x}{q^{i}}\right)_{1} c_{0}^{\prime}, \\
& D_{3}(x)=\frac{\psi(x)}{A(x)} R_{3}(x)=: \frac{\psi(x)}{A(x)\left(b_{1}\right)_{1}} \prod_{i=0}^{m+n-1}\left(\frac{x}{q^{i}}\right)_{1} c_{0}^{\prime}\left(b_{1} x\right)_{1} \varphi(x),
\end{aligned}
$$

where

$$
\begin{aligned}
& R_{1}(x)=B(x) P(x) Q(q x)-A(x) P(q x) Q(x), \\
& R_{2}(x)=\left(a_{1}, b_{1} x\right)_{1} P(x) \bar{Q}(x)-\left(a_{1} x, b_{1}\right)_{1} \bar{P}(x) Q(x), \\
& R_{3}(x)=\left(a_{1}, b_{1} x\right)_{1} A_{1}(x) P(q x) \bar{Q}(x)-\left(b_{1}\right)_{1} B(x) \bar{P}(x) Q(q x) .
\end{aligned}
$$

Here $c_{0}$ and $c_{0}^{\prime}$ are some constants depending on the parameters $a_{i}, b_{j}, m$ and $n$. Computing Taylor expansions at $x=0$ and $x=\infty$ in the expressions $R_{2}(x)$ and $R_{3}(x)$ (A.8), we determine $\varphi$ by (5.9). As a result, we obtain the desired relations $L_{2}$ and $L_{3}$ (A.3) where $C_{0}=\frac{c_{0}}{c_{0}^{\prime}}$ and $C_{1}=\frac{\left(a_{1}\right)_{1} \bar{c}_{0}}{c_{0}^{\prime}}$.

Next we have:

Proposition A.2. The constants $f$ and $g$ satisfy the q-Painlevé type system (5.8), and they play the role of dependent variables for (5.8).

Proof. The compatibility of the relations (A.3) gives the system (5.8).

\section{A.2 Particular solution}

We construct particular solutions of the $q$-Painlevé type system (2.2) given in terms of the $q$ hypergeometric function ${ }_{4} \varphi_{3}$ in Section 5.2. We derive the explicit forms (5.7) of variables $\{f, g\}$ appearing in the Casorati determinants $D_{1}$ and $D_{3}$ (A.7). They are interpreted as the particular solutions for the system (5.8), due to Proposition A.2.

Proposition A.3 ([7], see also [6, 24, 28]). For a given sequence $\psi_{s}$, the polynomials $P(x)$ and $Q(x)$ of degree $m$ and $n$ satisfying a Padé interpolation problem

$$
\psi_{s}=\frac{P\left(x_{s}\right)}{Q\left(x_{s}\right)}, \quad s=0,1, \ldots, m+n,
$$


is given as the following determinant expressions:

$$
\begin{aligned}
& P(x)=\mathcal{F}(x) \operatorname{det}\left[\sum_{s=0}^{m+n} u_{s} \frac{x_{s}^{i+j}}{x-x_{s}}\right]_{i, j=0}^{n}, \\
& Q(x)=\operatorname{det}\left[\sum_{s=0}^{m+n} u_{s} x_{s}^{i+j}\left(x-x_{s}\right)\right]_{i, j=0}^{n-1},
\end{aligned}
$$

where $u_{s}=\frac{\psi_{s}}{\mathcal{F}^{\prime}\left(x_{s}\right)}$ and $\mathcal{F}(x)=\prod_{i=0}^{m+n}\left(x-x_{i}\right)$.

Proposition A.4 ([6, 24, 28]). In the q-grid case of the problem (A.9) (i.e., interpolation points $x_{s}=q^{s}$ ), the formula (A.10) takes the following form:

$$
\begin{aligned}
& P(x)=\frac{\mathcal{F}(x)}{(q)_{m+n}^{n+1}} \operatorname{det}\left[\sum_{s=0}^{m+n} \psi_{s} \frac{\left(q^{-(m+n)}\right)_{s}}{(q)_{s}} \frac{q^{s(i+j+1)}}{x-q^{s}}\right]_{i, j=0}^{n}, \\
& Q(x)=\frac{1}{(q)_{m+n}^{n}} \operatorname{det}\left[\sum_{s=0}^{m+n} \psi_{s} \frac{\left(q^{-(m+n)}\right)_{s}}{(q)_{s}} q^{s(i+j+1)}\left(x-q^{s}\right)\right]_{i, j=0}^{n-1} .
\end{aligned}
$$

Proof. Substituting the expressions

$$
\mathcal{F}(x)=\prod_{s=0}^{m+n}\left(x-q^{s}\right), \quad \mathcal{F}^{\prime}\left(x_{s}\right)=\frac{(q)_{s}(q)_{m+n}}{q^{s}\left(q^{-(m+n)}\right)_{s}},
$$

into the formula (A.10), then we obtain the desired form (A.11).

Remark A.5. The normalization of the polynomials $P(x)$ and $Q(x)$ expressed in the formulas (A.10) and (A.11) differ from the convention $P(0)=1$ as fixed beneath the interpolation condition (A.2). This difference does not influence the result in Proposition A.7, because the common normalization factors of $P$ and $Q$ cancel in (A.14) and (A.15).

Proposition A.6. The polynomials $P(x)$ and $Q(x)$ defined in Section A.1 have the following particular values:

$$
\begin{array}{ll}
P\left(\frac{1}{a_{s}}\right)=\frac{\left(a_{s}\right)_{m+n+1}}{a_{s}^{m}\left(a_{s}\right)_{1}^{n+1}(q)_{m+n}^{n+1}} T_{a_{s}}\left(\tau_{m, n}\right), & Q\left(\frac{q}{a_{s}}\right)=\frac{q^{n}\left(\frac{a_{s}}{q}\right)_{1}^{n}}{a_{s}^{n}(q)_{m+n}^{n}} T_{a_{s}}^{-1}\left(\tau_{m+1, n-1}\right), \\
P\left(\frac{q}{b_{s}}\right)=\frac{q^{m}\left(\frac{b_{s}}{q}\right)_{m+n+1}}{b_{s}^{m}\left(\frac{b_{s}}{q}\right)_{1}^{n+1}(q)_{m+n}^{n+1}} T_{b_{s}}^{-1}\left(\tau_{m, n}\right), & Q\left(\frac{1}{b_{s}}\right)=\frac{\left(b_{s}\right)_{1}^{n}}{b_{s}^{n}(q)_{m+n}^{n}} T_{b_{s}}\left(\tau_{m+1, n-1}\right),
\end{array}
$$

for $s=1,2,3$. Here $\tau_{m, n}$ is defined by (5.4).

Proof. This proof follows from the formula (A.11) and the sequence $\psi_{s}=\psi\left(q^{s}\right)=\prod_{i=1}^{3} \frac{\left(b_{i}\right)_{s}}{\left(a_{i}\right)_{s}}$.

Proposition A.7. The particular values of $f$ and $g$ determined by (5.7) give particular solutions of the system (5.8).

Proof. From the first equation of (A.7), we have

$$
\frac{1-\frac{f}{a_{i}}}{1-\frac{f}{b_{j}}}=-\frac{a_{i}}{b_{j}} \prod_{s=0}^{m+n-1} \frac{\left(\frac{1}{b_{j} q^{s}}\right)_{1}}{\left(\frac{1}{a_{i} q^{s}}\right)} \frac{B\left(\frac{1}{a_{i}}\right)}{A\left(\frac{1}{b_{j}}\right)} \frac{P\left(\frac{1}{a_{i}}\right) Q\left(\frac{q}{a_{i}}\right)}{P\left(\frac{q}{b_{j}}\right) Q\left(\frac{1}{b_{j}}\right)}, \quad i, j=1,2,3,
$$


where $A$ and $B$ are as in Appendix A.1. From the second and third equations of (A.7), we have

$$
\begin{aligned}
& 1+\frac{g}{a_{i}}+\frac{\kappa}{a_{i}^{2}}=-\frac{\prod_{s=0}^{m+n}\left(\frac{1}{a_{1} q^{s}}\right)_{1}}{\prod_{s=0}^{m+n-1}\left(\frac{1}{a_{i} q^{s}}\right)_{1}} \frac{\left(b_{1}\right)_{1} B_{1}\left(\frac{1}{a_{i}}\right)}{\left(a_{1}, \frac{b_{1}}{a_{1}}\right)_{1}} \frac{\bar{P}\left(\frac{1}{a_{i}}\right) Q\left(\frac{q}{a_{i}}\right)}{P\left(\frac{1}{a_{1}}\right) \bar{Q}\left(\frac{1}{a_{1}}\right)}, \quad i=2,3, \\
& 1+\frac{g}{b_{i}}+\frac{\kappa}{b_{i}^{2}}=-\frac{\prod_{s=0}^{m+n}\left(\frac{1}{b_{1} q^{s}}\right)_{1}}{\prod_{s=0}^{m+n-1}\left(\frac{1}{b_{i} q^{s}}\right)_{1}} \frac{\left(a_{1}\right)_{1} A_{1}\left(\frac{1}{b_{i}}\right)}{\left(\frac{a_{1}}{b_{1}}, b_{1}\right)_{1}} \frac{P\left(\frac{q}{b_{i}}\right) \bar{Q}\left(\frac{1}{b_{i}}\right)}{\bar{P}\left(\frac{1}{b_{1}}\right) Q\left(\frac{1}{b_{1}}\right)}, \quad i=2,3,
\end{aligned}
$$

where $A_{1}$ and $B_{1}$ are as in Appendix A.1. Substituting the particular values (A.13) into the expressions (A.14) and (A.15) respectively, we obtain the desired particular solutions (5.7).

\section{Acknowledgements}

The author shall be thankful to Professor Yasuhiko Yamada for valuable discussions. The author is also grateful to the referees for stimulating comments. This work was partially supported by Expenses Revitalizing Education and Research of Akashi College (0217030).

\section{References}

[1] Dzhamay A., Sakai H., Takenawa T., Discrete Schlesinger transformations, their Hamiltonian formulation, and difference Painlevé equations, arXiv:1302.2972.

[2] Dzhamay A., Takenawa T., Geometric analysis of reductions from Schlesinger transformations to difference Painlevé equations, in Algebraic and Analytic Aspects of Integrable Systems and Painlevé Equations, Contemp. Math., Vol. 651, Amer. Math. Soc., Providence, RI, 2015, 87-124, arXiv:1408.3778.

[3] Gasper G., Rahman M., Basic hypergeometric series, Encyclopedia of Mathematics and its Applications, Vol. 96, 2nd ed., Cambridge University Press, Cambridge, 2004.

[4] Grammaticos B., Ohta Y., Ramani A., Sakai H., Degeneration through coalescence of the $q$-Painlevé VI equation, J. Phys. A: Math. Gen. 31 (1998), 3545-3558.

[5] Grammaticos B., Ramani A., On a novel q-discrete analogue of the Painlevé VI equation, Phys. Lett. A 257 (1999), 288-292.

[6] Ikawa Y., Hypergeometric solutions for the $q$-Painlevé equation of type $E_{6}^{(1)}$ by the Padé method, Lett. Math. Phys. 103 (2013), 743-763, arXiv:1207.6446.

[7] Jacobi C.G.J., Über die Darstellung einer Reihe gegebner Werthe durch eine gebrochne rationale Function, J. Reine Angew. Math. 30 (1846), 127-156.

[8] Jimbo M., Miwa T., Ueno K., Monodromy preserving deformation of linear ordinary differential equations with rational coefficients. I. General theory and $\tau$-function, Phys. D 2 (1981), 306-352.

[9] Jimbo M., Miwa T., Monodromy preserving deformation of linear ordinary differential equations with rational coefficients. II, Phys. D 2 (1981), 407-448.

[10] Jimbo M., Miwa T., Monodromy preserving deformation of linear ordinary differential equations with rational coefficients. III, Phys. D 4 (1981), 26-46.

[11] Jimbo M., Sakai H., A q-analog of the sixth Painlevé equation, Lett. Math. Phys. 38 (1996), 145-154.

[12] Kajiwara K., Masuda T., Noumi M., Ohta Y., Yamada Y., ${ }_{10} E_{9}$ solution to the elliptic Painlevé equation, J. Phys. A: Math. Gen. 36 (2003), L263-L272, nlin.SI/0303032.

[13] Kajiwara K., Masuda T., Noumi M., Ohta Y., Yamada Y., Hypergeometric solutions to the q-Painlevé equations, Int. Math. Res. Not. 2004 (2004), 2497-2521, nlin.SI/0403036.

[14] Kajiwara K., Masuda T., Noumi M., Ohta Y., Yamada Y., Construction of hypergeometric solutions to the q-Painlevé equations, Int. Math. Res. Not. 2005 (2005), 1441-1463, nlin.SI/0501051. 
[15] Kajiwara K., Noumi M., Yamada Y., q-Painlevé systems arising from q-KP hierarchy, Lett. Math. Phys. 62 (2002), 259-268, nlin.SI/0112045.

[16] Kajiwara K., Noumi M., Yamada Y., Geometric aspects of Painlevé equations, J. Phys. A: Math. Theor. 50 (2017), 073001, 164 pages, arXiv:1509.08186.

[17] Knuth D.E., Two notes on notation, Amer. Math. Monthly 99 (1992), 403-422.

[18] Mano T., Determinant formula for solutions of the Garnier system and Padé approximation, J. Phys. A: Math. Theor. 45 (2012), 135206, 14 pages.

[19] Mano T., Tsuda T., Two approximation problems by Hermite and the Schlesinger transformations, in Novel Development of Nonlinear Discrete Integrable Systems, RIMS Kôkyûroku Bessatsu, Vol. B47, Res. Inst. Math. Sci. (RIMS), Kyoto, 2014, 77-86.

[20] Mano T., Tsuda T., Hermite-Padé approximation, isomonodromic deformation and hypergeometric integral, Math. Z. 285 (2017), 397-431, arXiv:1502.06695.

[21] Masuda T., Hypergeometric $\tau$-functions of the $q$-Painlevé system of type $E_{7}^{(1)}$, SIGMA 5 (2009), 035, 30 pages, arXiv:0903.4102.

[22] Masuda T., Hypergeometric $\tau$-functions of the $q$-Painlevé system of type $E_{8}^{(1)}$, Ramanujan J. 24 (2011), $1-31$.

[23] Murata M., Lax forms of the q-Painlevé equations, J. Phys. A: Math. Theor. 42 (2009), 115201, 17 pages, arXiv:0810.0058.

[24] Nagao H., The Padé interpolation method applied to q-Painlevé equations, Lett. Math. Phys. 105 (2015), 503-521, arXiv:1409.3932.

[25] Nagao H., The Padé interpolation method applied to $q$-Painlevé equations II (differential grid version), Lett. Math. Phys. 107 (2017), 107-127, arXiv:1509.05892.

[26] Nagao H., Lax pairs for additive difference Painlevé equations, arXiv:1604.02530.

[27] Nagao H., Hypergeometric special solutions for $d$-Painlevé equations, arXiv:1706.10101.

[28] Nagao H., Yamada Y., Study of $q$-Garnier system by Padé method, arXiv:1601.01099.

[29] Nagao H., Yamada Y., Variations of $q$-Garnier system, arXiv:1710.03998.

[30] Noumi M., Tsujimoto S., Yamada Y., Padé interpolation for elliptic Painlevé equation, in Symmetries, Integrable Systems and Representations, Springer Proc. Math. Stat., Vol. 40, Springer, Heidelberg, 2013, 463-482, arXiv:1204.0294.

[31] Ohta Y., Ramani A., Grammaticos B., An affine Weyl group approach to the eight-parameter discrete Painlevé equation, J. Phys. A: Math. Gen. 34 (2001), 10523-10532.

[32] Ohyama Y., Kawamuko H., Sakai H., Okamoto K., Studies on the Painlevé equations. V. Third Painlevé equations of special type $P_{\mathrm{III}}\left(D_{7}\right)$ and $P_{\mathrm{III}}\left(D_{8}\right)$, J. Math. Sci. Univ. Tokyo 13 (2006), 145-204.

[33] Ohyama Y., Okumura S., A coalescent diagram of the Painlevé equations from the viewpoint of isomonodromic deformations, J. Phys. A: Math. Gen. 39 (2006), 12129-12151, math.CA/0601614.

[34] Okamoto K., Sur les feuilletages associés aux équations du second ordre à points critiques fixes de P. Painlevé, Japan. J. Math. (N.S.) 5 (1979), 1-79.

[35] Ormerod C.M., Rains E.M., Commutation relations and discrete Garnier systems, SIGMA 12 (2016), 110, 50 pages, arXiv:1601.06179.

[36] Ormerod C.M., Rains E.M., An elliptic Garnier system, Comm. Math. Phys. 355 (2017), 741-766, arXiv:1607.07831.

[37] Rains E.M., An isomonodromy interpretation of the hypergeometric solution of the elliptic Painlevé equation (and generalizations), SIGMA 7 (2011), 088, 24 pages, arXiv:0807.0258.

[38] Ramani A., Grammaticos B., Tamizhmani T., Tamizhmani K.M., Special function solutions of the discrete Painlevé equations, Comput. Math. Appl. 42 (2001), 603-614.

[39] Sakai H., Rational surfaces associated with affine root systems and geometry of the Painlevé equations, Comm. Math. Phys. 220 (2001), 165-229.

[40] Sakai H., Hypergeometric solution of q-Schlesinger system of rank two, Lett. Math. Phys. 73 (2005), 237-247.

[41] Sakai H., A q-analog of the Garnier system, Funkcial. Ekvac. 48 (2005), 273-297.

[42] Sakai H., Lax form of the $q$-Painlevé equation associated with the $A_{2}^{(1)}$ surface, J. Phys. A: Math. Gen. 39 (2006), 12203-12210. 
[43] Suzuki T., A $q$-analogue of the Drinfeld-Sokolov hierarchy of type $A$ and $q$-Painlevé system, in Algebraic and Analytic Aspects of Integrable Systems and Painlevé Equations, Contemp. Math., Vol. 651, Amer. Math. Soc., Providence, RI, 2015, 25-38, arXiv:1105.4240.

[44] Suzuki T., A reformulation of the generalized $q$-Painlevé VI system with $W\left(A_{2 n+1}^{(1)}\right)$ symmetry, J. Integrable Syst. 2 (2017), xyw017, 18 pages, arXiv:1602.01573.

[45] Takenawa T., Weyl group symmetry of type $D_{5}^{(1)}$ in the $q$-Painlevé V equation, Funkcial. Ekvac. 46 (2003), 173-186.

[46] Yamada Y., A Lax formalism for the elliptic difference Painlevé equation, SIGMA 5 (2009), 042, 15 pages, arXiv:0811.1796.

[47] Yamada Y., Padé method to Painlevé equations, Funkcial. Ekvac. 52 (2009), 83-92.

[48] Yamada Y., Lax formalism for $q$-Painlevé equations with affine Weyl group symmetry of type $E_{n}^{(1)}$, Int. Math. Res. Not. 2011 (2011), 3823-3838, arXiv:1004.1687.

[49] Yamada Y., A simple expression for discrete Painlevé equations, in Novel Development of Nonlinear Discrete Integrable Systems, RIMS Kôkyûroku Bessatsu, Vol. B47, Res. Inst. Math. Sci. (RIMS), Kyoto, 2014, 87-95.

[50] Yamada Y., An elliptic Garnier system from interpolation, SIGMA 13 (2017), 069, 8 pages, arXiv:1706.05155. 